\title{
On the stability of highly-charged Reissner-Nordström black holes to charged scalar perturbations
}

\author{
Shahar Hod \\ The Ruppin Academic Center, Emeq Hefer 40250, Israel \\ and \\ The Hadassah Institute, Jerusalem 91010, Israel
}

(Dated: July 5, 2018)

\begin{abstract}
The stability of Reissner-Nordström black holes under the influence of neutral perturbation fields was proved by Moncrief four decades ago. However, the superradiant scattering phenomenon, which characterizes the dynamics of charged bosonic fields in these charged black-hole spacetimes, imposes a greater and non-trivial threat on their stability. According to this well-known phenomenon, integer-spin charged fields interacting with a Reissner-Nordström black hole can be amplified (gain energy) by extracting some of the black-hole Coulomb energy. If, in addition to being electrically charged, the incident bosonic fields also possess non-zero rest masses, then the mutual gravitational attraction between the central black hole and the fields may prevent the extracted energy and electric charge from escaping to infinity. One may suspect that the physical mechanism of superradiant amplification of charged bosonic fields in the charged Reissner-Nordström black-hole spacetime, when combined with the confinement mechanism provided by the mutual gravitational attraction between the black hole and the massive fields, may lead to a superradiant instability of the ReissnerNordström black-hole spacetime. (This suspicion is mainly based on our experience with rotating Kerr black holes, which are known to be characterized by an analogous superradiant instability when coupled to massive bosonic fields). However, in this paper we show that, for highly-charged ReissnerNordström black holes in the charge interval $8 / 9<(Q / M)^{2}<1$, the two physical mechanisms which are required in order to trigger the superradiant instability phenomenon in the black-hole spacetime [namely: (1) the superradiant amplification of incident charged scalar fields by the charged black hole, and (2) the existence of a binding potential well in the black-hole exterior region which prevents the extracted energy and electric charge from escaping to infinity] cannot operate simultaneously. In particular, we shall prove that, in the superradiant regime, there is no trapping potential well in the black-hole exterior region. Our stability results for the highly-charged Reissner-Nordström black holes in the regime $8 / 9<(Q / M)^{2}<1$, combined with former analytical studies which explored the stability of these charged black holes in the complementary regime $(Q / M)^{2} \leq 8 / 9$, establish the fact that charged Reissner-Nordström black holes are stable to charged massive scalar perturbations in the entire parameter space.
\end{abstract}

\section{INTRODUCTION}

Rotating Kerr black holes are known to be super-radiantly unstable under the influence of co-rotating massive bosonic fields [1 4]. It has recently been shown that, for a scalar field of mass $\mu$ and azimuthal harmonic index $m$, the instability regime is bounded by the relation [5-7]

$$
0<\mu<m \Omega_{\mathrm{H}}
$$

where $\Omega_{\mathrm{H}}$ is the angular velocity of the black-hole horizon [8].

The instability of the composed Kerr-massive-bosonic-field system is a direct consequence of the superradiance phenomenon which characterizes the dynamics of integer-spin fields in spinning black-hole spacetimes [1 - 4, 9]. In particular, it is well established that a bosonic field mode of frequency $\omega$ impinging upon a spinning Kerr black hole is amplified (extracts rotational energy and angular momentum from the black hole) if the superradiance condition $[1,2]$

$$
\omega<m \Omega_{\mathrm{H}}
$$

is satisfied.

While the physical mechanism of superradiant amplification [1-4, [9] is required in order to extracts rotational energy from the spinning Kerr black hole, an additional physical mechanism is required in order to trigger a genuine exponentially growing instability in the black-hole spacetime. In particular, for the instability to exist, one should prevent the bosonic fields from radiating their energy to infinity. For the case of massive bosonic fields propagating in the black-hole spacetime, it is the attractive gravitational interaction between the central black hole and the massive fields which provides a natural confinement mechanism, thus preventing the extracted rotational energy from escaping 
to infinity. In particular, it is well established [1-4] that field modes whose conserved frequencies are bounded from above by

$$
\omega^{2}<\mu^{2}
$$

are prevented from escaping to infinity [see Eq. (15) below].

A similar physical mechanism, which involves the superradiant amplification of charged bosonic fields, threatens the stability of charged Reissner-Nordström black holes. In this case, the superradiant scattering phenomenon (that is, the amplification of incident charged bosonic fields) occurs for frequencies in the regime [10]

$$
\omega<q \Phi_{\mathrm{H}}
$$

where $q$ and $\Phi_{\mathrm{H}}=Q / r_{+}$are respectively the charge coupling constant of the incident scalar field and the electric potential of the central Reissner-Nordström black hole. (Here $Q$ and $r_{+}$are respectively the electric charge and horizon radius of the black hole [11]).

The scattering of charged bosonic fields in the superradiant regime (4) is characterized by the extraction of Coulomb energy and electric charge from the charged Reissner-Nordström (RN) black hole [10]. It is thus natural to suspect that the superradiant phenomenon, which characterizes the dynamics of charged massive bosonic fields in the charged RN black-hole spacetime, together with the natural confinement mechanism (3) provided by the attractive gravitational interaction between the central black hole and the massive fields, may lead to an instability of the charged RN spacetime [12.

The suspicion that the charged RN black-hole spacetime may be unstable to charged massive scalar perturbations is based on the similarity between the physical phenomenon of superradiant amplification of charged bosonic fields in the charged RN black-hole spacetime and the physical phenomenon of superradiant amplification of rotating massive bosonic fields in the rotating Kerr black-hole spacetime. As discussed above, in the later case the superradiant amplification of massive scalar perturbations is known to produce exponentially growing instabilities in the Kerr black-hole spacetime.

However, in [13] we have proved that charged RN black holes in the regime

$$
(Q / M)^{2} \leq \frac{8}{9}
$$

are actually stable under the influence of charged scalar perturbations [14]. The main goal of the present paper is to analyze the stability under charged scalar perturbations of highly-charged RN black holes in the complementary regime

$$
\frac{8}{9}<(Q / M)^{2}<1
$$

Below we shall provide evidence for the stability of the composed RN-charged-scalar-field system in the regime (6). In particular, we shall show that, for highly-charged RN black holes in the regime (6), the two physical mechanisms which are required in order to trigger the superradiant instability phenomenon [namely: (1) the superradiant amplification of the charged bosonic fields, and (2) the existence of a binding potential well in the black-hole exterior region which prevents the extracted energy and electric charge from escaping to infinity] cannot operate simultaneously. Our results thus support the stability of these highly-charged RN black holes.

\section{DESCRIPTION OF THE SYSTEM}

The physical system we shall analyze is composed of a massive charged scalar field which is linearly coupled to a charged Reissner-Nordström black hole. The RN black-hole spacetime is described by the line element [15]

$$
d s^{2}=-\frac{\Delta(r)}{r^{2}} d t^{2}+\frac{r^{2}}{\Delta(r)} d r^{2}+r^{2}\left(d \theta^{2}+\sin ^{2} \theta d \phi^{2}\right),
$$

where [7]

$$
\Delta \equiv r^{2}-2 M r+Q^{2} .
$$

Here $M$ and $Q$ are respectively the mass and electric charge of the black hole, and $r$ is the radial areal coordinate. The black-hole (event and inner) horizons are determined by the zeroes of $\Delta(r)$ :

$$
r_{ \pm}=M \pm \sqrt{M^{2}-Q^{2}}
$$


The dynamics of the charged massive scalar field $\Psi$ in the charged RN black-hole spacetime is described by the Klein-Gordon wave equation [16 19]

$$
\left[\left(\nabla^{\nu}-i q A^{\nu}\right)\left(\nabla_{\nu}-i q A_{\nu}\right)-\mu^{2}\right] \Psi=0 .
$$

Here $\mu$ and $q$ are respectively the mass and charge coupling constant of the field [20], and $A_{\nu}=-\delta_{\nu}^{0} Q / r$ is the electromagnetic potential of the black hole. It proves useful to decompose the scalar field in the form

$$
\Psi(t, r, \theta, \phi)=\sum_{l m} e^{i m \phi} S_{l m}(\theta) \Delta^{-1 / 2} \psi_{l m}(r) e^{-i \omega t},
$$

where $\omega$ is the conserved frequency of the field mode, and $\{l, m\}$ are respectively the spherical harmonic index and the azimuthal harmonic index of the mode [21]. Remembering that any instability must set in via a real-frequency mode 2-4, 22], we shall consider here marginally-stable field modes with $\omega_{I}=0$.

Substituting the field decomposition (11) into the Klein-Gordon wave equation (10), one finds that the radial function $\psi$ and the angular function $S$ are determined by two ordinary differential equations which are coupled by a separation constant $K_{l}=l(l+1)$ 23 25]. The radial equation for $\psi$ takes the form of a Schrödinger-like wave equation [13, 16 -18]

$$
\frac{d^{2} \psi}{d r^{2}}+\left(\omega^{2}-V\right) \psi=0
$$

where

$$
\omega^{2}-V \equiv \frac{U+M^{2}-Q^{2}}{\Delta^{2}}
$$

with

$$
U \equiv\left(\omega r^{2}-q Q r\right)^{2}-\Delta\left[\mu^{2} r^{2}+l(l+1)\right] .
$$

Note that the bound-state resonances of the scalar field in the black-hole spacetime are characterized by exponentially decaying eigenfunctions at large distances from the black hole $[3,4]$ :

$$
\psi(r \rightarrow \infty) \sim r e^{-\sqrt{\mu^{2}-\omega^{2}} r} \quad \text { with } \quad \omega^{2}<\mu^{2} .
$$

\section{NO BOUND-STATE RESONANCES IN THE SUPERRADIANT REGIME}

As discussed in the Introduction, two distinct physical mechanisms are required to operate simultaneously in order to trigger a superradiant instability in the black-hole spacetime:

- The extraction of Coulomb energy from the charged black hole due to the physical mechanism of superradiant amplification of charged bosonic fields in the superradiant regime $\omega<q Q / r_{+}$[see Eq. (4)].

- The existence of a binding potential well in the black-hole exterior region which prevents the extracted energy from escaping to infinity. This trapping potential is required in order to support the bound-state resonances of the charged scalar fields in the black-hole exterior region. In particular, in order to support these bound-state scalar configurations, the potential well must be separated from the black-hole horizon by a potential barrier.

We shall prove below that these two physical mechanisms (that is, superradiant amplification and trapping of the fields) cannot operate simultaneously in the regime (6) of highly-charged RN black holes.

Specifically, we shall prove below that, in the superradiant regime (4), there are no bound-state resonances of the charged scalar fields in the black-hole exterior region [that is, we shall prove that, for highly-charged RN black holes in the regime (6), there are no trapping potential wells in the black-hole exterior region which are separated from the black-hole horizon by a potential barrier]. To that end, we shall now study the properties of the effective potential $V(r ; M, Q, \mu, q, \omega, l)$ that appears in the Schrödinger-like radial wave equation (12). 


\section{A. The gradient of the effective radial potential}

The gradient of the effective radial potential $V(r)$ can be expressed as a 4 th order polynomial function 13 ]

$$
-\frac{\Delta^{3}}{2} V^{\prime}(z ; M, Q, \mu, q, \omega, l)=a z^{4}+b z^{3}+c z^{2}+d z+e,
$$

where

$$
z \equiv r-r_{-}
$$

The expansion coefficients in (16) are given by [26]

$$
\begin{gathered}
a=M \mu^{2}+Q q \omega-2 M \omega^{2}, \\
b=\left(4 M r_{-}-2 M^{2}-Q^{2}\right) \mu^{2}+\left(-8 M r_{-}+2 Q^{2}\right) \omega^{2}+2 Q\left(M+2 r_{-}\right) q \omega-Q^{2} q^{2}+l(l+1), \\
c=-3 r_{-}^{2}\left(r_{+}-M\right) \mu^{2}+3 r_{-}^{3}\left(\frac{q Q}{r_{-}}-\omega\right)\left(2 \omega-\frac{q Q}{r_{-}}\right)-3\left(r_{+}-M\right) l(l+1),
\end{gathered}
$$

and

$$
e=2 r_{-}^{4}\left(r_{+}-M\right)\left(\omega-\frac{q Q}{r_{-}}\right)^{2}+2\left(r_{+}-M\right)^{3} .
$$

Note that the condition (3) for the existence of bound-state scalar resonances in the black-hole exterior region, together with the condition (4) for the existence of the superradiant amplification phenomenon of the trapped bosonic modes, imply that the mode frequency $\omega$ is restricted to the interval

$$
0 \leq \omega<\min \left\{\frac{q Q}{r_{+}}, \mu\right\} .
$$

\section{B. The signs of the coefficients $\{a, b, c, e\}$}

In order to analyze the spatial behavior of the effective radial potential $V(r)$, we shall first analyze the signs of the coefficients $\{a, b, c, e\}$ that appear in the gradient equation (16).

It was proved in 13] that, in the frequency interval (22), the coefficient $a$ is positive definite in the entire range $0 \leq(Q / M)^{2} \leq 1[27]$ :

$$
a>0
$$

In addition, inspection of Eq. (21) reveals that the coefficient $e$ is positive definite in the entire range $0 \leq(Q / M)^{2} \leq 1$ [13]:

$$
e>0
$$

We shall now prove that, for highly-charged Reissner-Nordström black holes in the charge interval [see Eq. (6)]

$$
\frac{8}{9}<x^{2}<1 \quad ; \quad x \equiv Q / M
$$

(at least) one of the inequalities

$$
b>0 \quad ; \quad c<0
$$

holds true. In particular, we shall henceforth assume that 28]

$$
c>0
$$


and prove that this inequality necessarily implies the inequality

$$
b>0
$$

in the frequency interval (22).

Note that a necessary condition for the validity of the inequality $c(\omega)>0$ is given by $\left(q Q / r_{-}-\omega\right)\left(2 \omega-q Q / r_{-}\right)>0$ [see Eq. (20)], or equivalently by $q Q / 2 r_{-}<\omega<q Q / r_{-}$. Taking cognizance of (22) and using the fact that $r_{-}<r_{+}$, one realizes that in the regime (27) the conserved mode frequency is bounded by [29]

$$
\frac{q Q}{2 r_{-}}<\omega<\min \left\{\frac{q Q}{r_{+}}, \mu\right\}
$$

There are two distinct cases which should be analyzed separately:

Case (A): Charged Reissner-Nordström black holes in the interval

$$
8 / 9<x^{2} \leq 4 \sqrt{3}-6
$$

This charge interval corresponds to the inequality $4 M r_{-}-2 M^{2}-Q^{2} \leq 0$ for the coefficient of $\mu^{2}$ in (19), in which case one can obtain a lower bound on the value of the coefficient $b(\omega)$ by substituting into (19) the maximally allowed value of the mass parameter $\mu^{2}$. This value is given by the inequality [see Eq. (20) with $c>0$ ]

$$
0<3 r_{-}^{2}\left(r_{+}-M\right) \mu^{2}<3 r_{-}^{3}\left(\frac{q Q}{r_{-}}-\omega\right)\left(2 \omega-\frac{q Q}{r_{-}}\right)-3\left(r_{+}-M\right) l(l+1) .
$$

Substituting (31) into (19), one finds

$$
b>I(\omega ; x)+\frac{2 \sqrt{1-x^{2}}}{\left(1-\sqrt{1-x^{2}}\right)^{2}} \cdot l(l+1)
$$

where

$$
I(\omega ; x) \equiv \frac{2 x^{2}-4+4 \sqrt{1-x^{2}}}{\sqrt{1-x^{2}}} \cdot(M \omega)^{2}+\frac{x^{2}+2-6 \sqrt{1-x^{2}}}{\sqrt{1-x^{2}}} \cdot q Q M \omega+\frac{3 \sqrt{1-x^{2}}-1}{\sqrt{1-x^{2}}\left(1-\sqrt{1-x^{2}}\right)} \cdot(q Q)^{2} .
$$

Note that $2 x^{2}-4+4 \sqrt{1-x^{2}}<0$ in the entire range $0<x^{2} \leq 1$, which implies that the dependence of $I(\omega)$ on $\omega$ is in the form of a convex parabola. Thus, $I(\omega)$ is minimized at the boundaries of the frequency interval (29). Substituting $\omega \rightarrow q Q / 2 r_{-}$into (33), one finds

$$
I\left(\omega \rightarrow q Q / 2 r_{-} ; x\right)=\frac{1}{2}(q Q)^{2}>0
$$

which obviously has a positive definite value. Substituting into (33) $\omega \rightarrow q Q / r_{+}$for the case $q Q / r_{+} \leq \mu$, one finds

$$
I\left(\omega \rightarrow q Q / r_{+} ; x\right)=\frac{16\left(1-\sqrt{1-x^{2}}\right)+2 x^{2}\left(5 \sqrt{1-x^{2}}-7\right)-x^{4}}{x^{4}} \cdot(q Q)^{2}>0
$$

where the inequality sign in (35) refers to RN black holes in the entire range $0 \leq x^{2} \leq 1$. This implies, in particular, that the expression (35) is a positive definite function in the charge interval (30). Substituting into (33) $\omega \rightarrow \mu$ for the case $q Q / 2 r_{-}<\mu<q Q / r_{+}[29]$, one finds [30]

$$
I(\omega \rightarrow \mu ; x)>\min \left\{I\left(\omega \rightarrow q Q / 2 r_{-} ; x\right), I\left(\omega \rightarrow q Q / r_{+} ; x\right)\right\}>0 .
$$

We therefore conclude that charged Reissner-Nordström black holes in the charge interval (30) are characterized by (at least) one of the relations [see Eqs. (27), (32), (34), (35), and (36)]

$$
b \geq I>0 \quad ; \quad c<0 .
$$

Case (B): Charged Reissner-Nordström black holes in the interval

$$
4 \sqrt{3}-6<x^{2}<1 .
$$


This charge interval corresponds to the inequality $4 M r_{-}-2 M^{2}-Q^{2}>0$ for the coefficient of $\mu^{2}$ in (19). Remembering that $\mu^{2}>\omega^{2}$ [see (15)], one can obtain a lower bound on the value of the coefficient $b(\omega)$ by replacing $\mu \rightarrow \omega$ in (19). One then finds

$$
b>J(\omega ; x)+l(l+1),
$$

where

$$
J(\omega ; x) \equiv\left(x^{2}+4 \sqrt{1-x^{2}}-6\right) \cdot(M \omega)^{2}+2\left(3-2 \sqrt{1-x^{2}}\right) \cdot q Q M \omega-(q Q)^{2} .
$$

Note that $x^{2}+4 \sqrt{1-x^{2}}-6<0$ in the entire range $0 \leq x^{2} \leq 1$, which implies that the dependence of $J(\omega)$ on $\omega$ is in the form of a convex parabola. Thus, $J(\omega)$ is minimized at the boundaries of the frequency interval (29). Substituting $\omega \rightarrow q Q / 2 r_{-}$into (40), one finds

$$
J\left(\omega \rightarrow q Q / 2 r_{-} ; x\right)=\frac{6-3 x^{2}-8 \sqrt{1-x^{2}}}{4\left(1-\sqrt{1-x^{2}}\right)^{2}} \cdot(q Q)^{2}>0,
$$

where the inequality sign in (41) refers to RN black holes in the regime $x^{2}>(8 \sqrt{7}-14) / 9$. In particular, one finds that the expression (41) is a positive definite function in the entire charge interval (38) [note that $(8 \sqrt{7}-14) / 9<4 \sqrt{3}-6$ ]. Substituting into (40) $\omega \rightarrow q Q / r_{+}$for the case $q Q / r_{+} \leq \mu$, one finds

$$
J\left(\omega \rightarrow q Q / r_{+} ; x\right)=\frac{6 x^{2}+4 \sqrt{1-x^{2}}-6}{\left(1+\sqrt{1-x^{2}}\right)^{2}} \cdot(q Q)^{2}>0,
$$

where the inequality sign in (42) refers to RN black holes in the regime $5 / 9<x^{2}<1$. In particular, one finds that the expression (42) is a positive definite function in the entire charge interval (38) [note that $5 / 9<4 \sqrt{3}-6$ ]. Substituting into (40) $\omega \rightarrow \mu$ for the case $q Q / 2 r_{-}<\mu<q Q / r_{+}$[29], one finds [30]

$$
J(\omega \rightarrow \mu ; x)>\min \left\{J\left(\omega \rightarrow q Q / 2 r_{-} ; x\right), J\left(\omega \rightarrow q Q / r_{+} ; x\right)\right\}>0 .
$$

We therefore conclude that charged RN black holes in the charge interval (38) are characterized by (at least) one of the relations [see Eqs. (27), (39), (41), (42), and (43)]

$$
b \geq J>0 \quad ; \quad c<0 .
$$

\section{The spatial behavior of the effective radial potential}

We shall now analyze the spatial behavior of the effective radial potential $V(r)$ that appears in the Schrödinger-like radial equation (12). In particular, we shall determine the signs of the four roots $\left\{z_{1}, z_{2}, z_{3}, z_{4}\right\}$ which characterize the gradient equation [see Eq. (16)]

$$
V^{\prime}(z)=0 .
$$

Taking cognizance of the inequality (23), one finds [see Eq. (17)]

$$
V^{\prime}(r \rightarrow \infty) \rightarrow 0^{-}
$$

for the asymptotic behavior of the effective radial potential. In addition, from Eqs. (13) and (14) one finds that the effective radial potential $V(r)$ is characterized by the following two properties:

$$
V\left(r \rightarrow r_{+}\right) \rightarrow-\infty
$$

and

$$
V\left(r \rightarrow r_{-}\right) \rightarrow-\infty
$$

Taking cognizance of Eqs. (46) and (47), one concludes that the effective potential $V(r)$ is characterized by at least one maximum point in the exterior black-hole region $r>r_{+}$[13]. We denote that maximum point by $z_{4}$, where

$$
z_{4}>0
$$


In addition, taking cognizance of Eqs. (47) and (48), one concludes that the effective potential $V(r)$ has at least one maximum point which is located in the interval $r_{-}<r<r_{+}$between the two horizons of the charged black hole [13]. We denote that maximum point by $z_{3}$, where

$$
z_{4}>z_{3}>0
$$

Taking cognizance of Eqs. (23) and (24), and using the well-known relation

$$
z_{1} \cdot z_{2} \cdot z_{3} \cdot z_{4}=\frac{e}{a}
$$

for the four roots of a quartic equation, one finds

$$
z_{1} \cdot z_{2} \cdot z_{3} \cdot z_{4}>0
$$

Likewise, taking cognizance of Eqs. (23), (37), and (44), and using the well-known relations

$$
z_{1}+z_{2}+z_{3}+z_{4}=-\frac{b}{a}
$$

and

$$
z_{1} \cdot z_{2}+z_{1} \cdot z_{3}+z_{1} \cdot z_{4}+z_{2} \cdot z_{3}+z_{2} \cdot z_{4}+z_{3} \cdot z_{4}=\frac{c}{a}
$$

for the four roots of a quartic equation, one concludes that highly-charged RN black holes in the charge interval (6) are characterized by (at least) one of the following relations:

$$
z_{1}+z_{2}+z_{3}+z_{4}<0 \quad ; \quad z_{1} \cdot z_{2}+z_{1} \cdot z_{3}+z_{1} \cdot z_{4}+z_{2} \cdot z_{3}+z_{2} \cdot z_{4}+z_{3} \cdot z_{4}<0 .
$$

Finally, taking cognizance of Eqs. (50), (52), and (55), one concludes that $V^{\prime}(z)$ is characterized by two negative roots (we denote them by $z_{1}$ and $z_{2}$, where $\left.z_{1} \leq z_{2}<0\right)$ and two positive roots $\left(0<z_{3}<z_{4}\right)$.

Our analysis thus reveals that, for bound states of the scalar fields [characterized by $\omega^{2}<\mu^{2}$, see (15)] in the superradiant regime [characterized by $\omega<q Q / r_{+}$, see (4)], the gradient $V^{\prime}(z)$ of the effective potential that appears in the Schrödinger-like radial equation (12) is characterized by two positive roots and two negative roots. The physical root $z_{4}>0$ (or equivalently, $r_{4}>r_{+}$) corresponds to a maximum point of the effective radial potential $V(r)$ in the black-hole exterior region. The negative roots $\left\{z_{1}, z_{2}\right\}$ and the positive root $z_{3}$ correspond to three roots of $V^{\prime}(r)$ in the black-hole interior region $r<r_{+}$.

We have therefore proved that, in the superradiant regime (4), the effective radial potential $V(r)$ is characterized by the existence of only one maximum point (and no minima at all) in the black-hole exterior region. This implies that, in the superradiant regime (4), there is no binding potential well in the physical region $r>r_{+}$which is separated from the black-hole horizon by a potential barrier. One therefore concludes that, for highly-charged RN black holes in the charge interval $8 / 9<(Q / M)^{2}<1$, there are no bound-state resonances (with $\omega^{2}<\mu^{2}$ ) of the charged massive scalar fields in the superradiant regime $\omega<q Q / r_{+}$.

\section{SUMMARY}

Motivated by the well-known superradiant instability phenomenon which characterizes the composed Kerr-massivescalar-field system, we have explored here the possible existence of an analogous superradiant instability for highlycharged Reissner-Nordström black holes [in the regime $8 / 9<(Q / M)^{2}<1$ ] coupled to charged massive scalar fields. It was shown that the two physical mechanisms which are required to operate simultaneously in order to trigger the superradiant instability phenomenon in the black-hole spacetime [namely: (1) the superradiant amplification of the charged scalar fields by the charged black hole, and (2) the existence of a binding potential well in the black-hole exterior region which prevents the extracted energy and electric charge from escaping to infinity] cannot operate simultaneously. In particular, we have proved that RN black holes in the charge interval $8 / 9<(Q / M)^{2}<1$ cannot support bound-state resonance (with $\omega^{2}<\mu^{2}$ ) of charged massive scalar fields in the superradiant regime $\omega<q Q / r_{+}$. This fact suggests that the dynamics of charged massive scalar fields in these highly-charged RN black-hole spacetimes is expected to be stable.

The stability results presented in this paper for the highly-charged $\mathrm{RN}$ black holes in the regime $8 / 9<(Q / M)^{2}<1$, combined with former analytical studies which explored the stability of these charged black holes in the complementary 
regime $(Q / M)^{2} \leq 8 / 9[13,14]$, establish the fact that charged Reissner-Nordström black holes are immune against the superradiant instability phenomenon in the entire parameter space.

Finally, we would like to stress that the present analysis excludes the existence of exponentially growing instabilities (bound-state resonances) of the charged scalar fields in the charged Reissner-Nordström black-hole spacetime. However, it is worth noting that milder forms of instabilities could appear, in principle [31].

\section{ACKNOWLEDGMENTS}

This research is supported by the Carmel Science Foundation. I thank Yael Oren, Arbel M. Ongo, Ayelet B. Lata, and Alona B. Tea for helpful discussions.

[1] Ya. B. Zel'dovich, Pis‘ma Zh. Eksp. Teor. Fiz. 14, 270 (1971) [JETP Lett. 14, 180 (1971)]; Zh. Eksp. Teor. Fiz. 62, 2076 (1972) [Sov. Phys. JETP 35, 1085 (1972)]; A. V. Vilenkin, Phys. Lett. B 78, 301 (1978).

[2] W. H. Press and S. A. Teukolsky, Nature 238, 211 (1972); W. H. Press and S. A. Teukolsky, Astrophys. J. 185, 649 (1973).

[3] T. Damour, N. Deruelle and R. Ruffini, Lett. Nuovo Cimento 15, 257 (1976); T. M. Zouros and D. M. Eardley, Annals of physics 118, 139 (1979); S. Detweiler, Phys. Rev. D 22, 2323 (1980); H. Furuhashi and Y. Nambu, Prog. Theor. Phys. 112, 983 (2004); V. Cardoso and J. P. S. Lemos, Phys. Lett. B 621, 219 (2005); V. Cardoso and S. Yoshida, JHEP 0507:009 (2005); S. R. Dolan, Phys. Rev. D 76, 084001 (2007); S. Hod and O. Hod, Phys. Rev. D 81, Rapid communication 061502 (2010) arXiv:0910.0734; H. R. Beyer, J. Math. Phys. 52, 102502 (2011); Y. S. Myung, Phys. Rev. D 84, 024048 (2011); S. Hod, Phys. Lett. B 713, 505 (2012); J. P. Lee, JHEP 1201, 091 (2012); S. Hod, Phys. Lett. B 718, 1489 (2013) arXiv:1304.6474; R. Brito, V. Cardoso, and P. Pani, Phys. Rev. D 88, 023514 (2013); S. R. Dolan, Phys. Rev. D 87, 124026 (2013); H. Witek, V. Cardoso, A. Ishibashi, and U. Sperhake, Phys. Rev. D 87, 043513 (2013); V. Cardoso, Gen. Relativ. and Gravit. 45, 2079 (2013); J. C. Degollado and C. A. R. Herdeiro, Gen. Rel. Grav. 45, 2483 (2013); R. Li, The Euro. Phys. Journal C 73, 2274 (2013); S. J. Zhang, B. Wang, E. Abdalla, arXiv:1306.0932; H. Witek, arXiv:1307.1145. Y. S. Myung, Phys. Rev. D 88, 104017 (2013); R. Li, Phys. Rev. D 88, 127901 (2013); H. Okawa, H. Witek, and V. Cardoso, Phys. Rev. D 89, 104032 (2014); B. Arderucio, arXiv:1404.3421; M. O. P. Sampaio, C. Herdeiro, M. Wang, Phys. Rev. D 90, 064004 (2014); Y. Brihaye, C. Herdeiro, and E. Radu, Phys. Lett. B 739, 1 (2014); C. L. Benone, L. C. B. Crispino, C. Herdeiro, and E. Radu, arXiv:1409.1593 C. Herdeiro, E. Radu, and H. Runarsson, arXiv:1409.2877.

[4] S. Hod, Phys. Rev. D 86, 104026 (2012) arXiv:1211.3202; S. Hod, The Euro. Phys. Journal C 73, 2378 (2013) arXiv:1311.5298; S. Hod, Phys. Rev. D 90, 024051 (2014) arXiv:1406.1179; S. Hod, Phys. Lett. B 739, 196 (2014) arXiv:1411.2609; C. A. R. Herdeiro and E. Radu, Phys. Rev. Lett. 112, 221101 (2014); C. A. R. Herdeiro and E. Radu, Phys. Rev. D 89, 124018 (2014); C. A. R. Herdeiro and E. Radu, arXiv:1405.3696.

[5] S. Hod, Phys. Lett. B 708, 320 (2012) arXiv:1205.1872.

[6] S. Hod, Submitted for publication in Class. Quant. Grav. (2014).

[7] We use natural units in which $G=c=\hbar=1$.

[8] It has recently been shown [6] that the upper bound (1) on the instability regime (that is, the upper bound on the scalar field mass which can trigger the superradiant instability) can be approached arbitrarily close in the eikonal (large- $m$ ) limit [6].

[9] V. Cardoso, O. J. C. Dias, J. P. S. Lemos and S. Yoshida, Phys. Rev. D 70, 044039 (2004) [Erratum-ibid. D 70, 049903 (2004)]; J. C. Degollado, C. A. R. Herdeiro, and H. F. Rúnarsson, Phys. Rev. D 88, 063003 (2013); J. C. Degollado and C. A. R. Herdeiro, Phys. Rev. D 89, 063005 (2014); S. Hod, Phys. Rev. D 88, 064055 (2013) arXiv:1310.6101; R. Li, arXiv:1404.6309, S. Hod, Phys. Rev. D 88, 124007 (2013) arXiv:1405.1045; S. Hod, Phys. Lett. B 736, 398 (2014); R. Li and J. Zhao, The Euro. Phys. Journal C 743051 (2014); S. Hod, Phys. Lett. B 736, 398 (2014) arXiv:1412.6108; C. L. Benone, L. C. B. Crispino, C. Herdeiro, and E. Radu, arXiv:1412.7278,

[10] J. D. Bekenstein, Phys. Rev. D 7, 949 (1973).

[11] We shall assume, without loss of generality, that $Q \geq 0$.

[12] A superradiant instability which is expected to be analogous to the superradiant instability of the Kerr black hole described above.

[13] S. Hod, Phys. Lett. B 718, 1489 (2013) arXiv:1304.6474.

[14] The stability of extremal $(|Q|=M)$ charged RN black holes to charged scalar perturbations was proved in S. Hod, Phys. Lett. B 713, 505 (2012).

[15] S. Chandrasekhar, The Mathematical Theory of Black Holes, (Oxford University Press, New York, 1983).

[16] S. Hod and T. Piran, Phys. Rev. D 58, 024017 (1998) arXiv:gr-qc/9712041]; S. Hod and T. Piran, Phys. Rev. D 58, 024018 (1998) arXiv:gr-qc/9801001; S. Hod and T. Piran, Phys. Rev. D 58, 024019 (1998) arXiv:gr-qc/9801060.

[17] T. Hartman, W. Song, and A. Strominger, JHEP 1003:118 (2010).

[18] S. Hod, Class. Quant. Grav. 23, L23 (2006) arXiv:gr-qc/0511047.

[19] S. Hod, Phys. Lett. A 374, 2901 (2010) arXiv:1006.4439.

[20] Note that $\mu$ and $q$ stand for $\mu / \hbar$ and $q / \hbar$, respectively. Hence, these field parameters have the dimensions of (length) ${ }^{-1}$.

[21] We shall henceforth omit the harmonic indexes $l$ and $m$ for brevity.

[22] J. B. Hartle and D. C. Wilkins, Commun. Math. Phys. 38, 47 (1974). 
[23] A. Ronveaux, Heun's differential equations. (Oxford University Press, Oxford, UK, 1995).

[24] C. Flammer, Spheroidal Wave Functions (Stanford University Press, Stanford, 1957).

[25] M. Abramowitz and I. A. Stegun, Handbook of Mathematical Functions (Dover Publications, New York, 1970).

[26] The explicit form of the expansion coefficient $d$ is given in [13]. However, here we shall not need the explicit form of this coefficient.

[27] The argument for the positivity of the coefficient $a$ in the frequency interval (22) goes as follows [13]: from (18) one learns that the function $a(\omega)$ is in the form of a convex parabola. Hence, $a(\omega)$ is minimized at the boundaries of the frequency interval (22). Substituting $\omega=0$ into (18), one finds $a(\omega=0)=M \mu^{2}>0$. Substituting into (18) $\omega \rightarrow q Q / r_{+}$for the case $q Q / r_{+} \leq \mu$, one finds $a\left(\omega \rightarrow q Q / r_{+}\right)=M \mu^{2}+\frac{Q^{2} q^{2}}{r_{+}}\left(1-\frac{2 M}{r_{+}}\right)>0$. Substituting into (18) $\omega \rightarrow \mu$ for the case $\mu<q Q / r_{+}$, one finds $a(\omega \rightarrow \mu)=\mu(Q q-M \mu)>\mu^{2}\left(r_{+}-M\right) \geq 0$. One therefore finds that, in the frequency interval (22), the coefficient $a$ is positive definite in the entire range $0 \leq(Q / M)^{2} \leq 1$.

[28] It was proved in [13] that $c$ is negative definite for charged RN black holes in the regime $(Q / M)^{2} \leq 8 / 9$.

[29] Note that the two inequalities in (29) can only be satisfied in the regime $\mu>q Q / 2 r_{-}$.

[30] This inequality follows from the fact that $I(\omega ; Q / M)$ is in the form of a convex parabola. Thus, $I\left(x_{2} ; Q / M\right)>$ $\min \left\{I\left(x_{1} ; Q / M\right), I\left(x_{3} ; Q / M\right)\right\}>0$ for $x_{1}<x_{2}<x_{3}$.

[31] I would like to thank the anonymous referee for this valuable comment. 